\section{RMD Open}

Rheumatic \&

Musculoskeletal Diseases

\title{
One-year effects of glucocorticoids on bone density: a meta-analysis in cohorts on high and low-dose therapy
}

\author{
Willem F Lems, ${ }^{1}$ Merel M E Baak, ${ }^{1}$ Lilian H D van Tuyl, ${ }^{1}$ Mariëtte C Lodder, ${ }^{2}$ \\ Ben A C Dijkmans, ${ }^{1}$ Maarten Boers ${ }^{1,3}$
}

To cite: Lems WF, Baak MME, van Tuyl LHD, et al. One-year effects of glucocorticoids on bone density: a meta-analysis in cohorts on high and lowdose therapy. RMD Open 2016;2:e000313.

doi:10.1136/rmdopen-2016000313

- Prepublication history and additional material is available. To view please visit the journal (http://dx.doi.org/ 10.1136/rmdopen-2016000313)

Received 26 May 2016 Revised 13 August 2016 Accepted 15 August 2016

\section{(a) CrossMark}

${ }^{1}$ Amsterdam Rheumatology and immunology Center, VUmc, Amsterdam,

The Netherlands

2Department of

Rheumatology, Spaarne

Gasthuis, Haarlem,

The Netherlands

${ }^{3}$ Department of Epidemiology and Biostatistics, VU

University Medical Center,

Amsterdam, The Netherlands

Correspondence to

Professor Maarten Boers;

eb@vumc.nl

\section{ABSTRACT}

Background: Bone loss during glucocorticoid (GC) therapy is poorly quantified.

Objective: Quantification of bone loss in GC-treated patients with chronic inflammatory diseases (CID; low dose) and transplants (high dose).

Methods: Meta-analysis of cohorts: PubMed, Cochrane, EMBASE and bibliographic searches (19952012). Eligible studies prospectively included GCtreated patients with two dual $\mathrm{X}$-ray absorptiometry measurements of spine or hip over a period of at least 12 months. Only supplementation with calcium or vitamin D3 was allowed. 5602 titles yielded 285 articles: 51 study arms in CID ( $\mathrm{N}=1565), 18$ study arms in transplantation ( $\mathrm{N}=571$ ). Prednisone-equivalent GC doses and inverse variance weighted mean bone changes were used in a random effects model.

Results: In CID, the mean GC dose was $8.7 \mathrm{mg} /$ day (range 1.2-16.4). The mean 1-year bone loss in the lumbar spine was $-1.7 \%(95 \% \mathrm{Cl}-2.2 \%$ to $-1.2 \%)$; in the femoral neck: $-1.3(-1.8$ to -0.7$)$. In transplantation, the mean GC dose was $18.9 \mathrm{mg} /$ day (range 6.0-52.7). Bone loss in the lumbar spine was $-3.6 \%(-5.2 \%$ to $-2.0 \%)$; in the femoral neck: $-3.1 \%$ $(-5.1 \%$ to $-1.1 \%)$. Within the two groups, bone loss was not related to $\mathrm{GC}$ dose.

Conclusion: In CID, GC-related bone loss appears limited and manageable if current anti-osteoporotic strategies are fully implemented. In transplantation, and probably also other high-dose settings, bone loss is considerable and represents unmet need. The heterogeneity probably reflects the important influence of other factors, most notably the underlying disease and the efficacy of GC treatment.

\section{INTRODUCTION}

Chronic use of glucocorticoids (GC) is probably the most common cause of secondary osteoporosis. GC diminish bone mass through various mechanisms. They have a direct negative effect on bone: they interfere with osteoblast function by inhibiting the WnT signalling pathway, and induce apoptosis

\section{Key messages}

What is already known about this subject?

- Glucocorticoids can induce bone loss, but the extent is poorly quantified.

What does this study add?

- Exhaustive meta-analysis of prospective studies in high-dose and low-dose settings without bone protection.

- Limited bone loss in chronic inflammatory disease treated with low or medium doses; more extensive bone loss in transplantation set tings with high doses.

- Large heterogeneity of findings suggests that many factors besides glucocorticoids-most notably disease severity-influence the extent of bone loss.

How might this impact on clinical practice?

- Adequate bone protection can probably prevent bone loss in chronic inflammatory disease and strongly limit its extent in transplantation settings.

- Adequate treatment of the underlying disease (including glucocorticoids where indicated) is very important in the prevention of bone loss.

of osteoblasts, while upregulation of receptor activator of nuclear factor $\mathrm{\kappa} B$ ligand results in elevated bone resorption. ${ }^{1}$ In addition, GC impair intestinal calcium uptake and increase renal calcium excretion, leading to a tendency for secondary hyperparathyroidism; another indirect effect of GC on fracture risk is muscular weakness and increased risk of falls. ${ }^{2-4}$ As a consequence, GC treatment is also associated with an increased risk of vertebral and non-vertebral fractures. ${ }^{5}$

GC are used in many diseases, including rheumatoid arthritis (RA), polymyalgia rheumatica, inflammatory bowel disease, dermatological and chronic obstructive pulmonary disease. A critical point is that in many of 
these diseases the disease activity and severity of the underlying disorder is also associated with bone loss. Since patients with the highest disease activity are in the greatest need of GC, confounding by indication makes quantification of actual bone loss a complicated task. However, several studies have shown that the strong immunosuppressive effect of GC limits the net effect on bone loss. ${ }^{67}$ This has been documented in RA, ${ }^{8}{ }^{9}$ and comparable mechanisms can be assumed in other diseases. It is well known that bone loss occurs rapidly during the first few months of GC therapy, followed by a slower but continued loss with ongoing use, probably because of the use of a higher initial dosage of GC and a higher initial disease activity. ${ }^{11} 11$

International guidelines dictate the use of calcium and vitamin $\mathrm{D}$ in all patients who initiate GC treatment, ${ }^{12-14}$ with addition of bisphosphonates in high-risk patients, for example, elderly patients and patients treated with high-dose GC.

In patients undergoing organ transplantation, the initial GC dose is usually much higher than in patients treated for chronic diseases, especially in the first 6 months after transplantation. Remarkably, research on GC-related bone loss in transplantation is relatively sparse and, to the best of our knowledge, a meta-analysis on bone loss in this patient group has not yet been performed.

We investigated the amount of bone loss in two patient groups exposed to GC during a period of 12 months: patients undergoing organ transplantation (lung, heart, lung/heart, liver and kidney) and patients with various chronic inflammatory diseases, including RA, systemic lupus erythematosus, polymyalgia rheumatica, vasculitis, granulomatosis with polyangiitis and inflammatory bowel disease.

\section{METHODS}

\section{Protocol and registration}

The analysis protocol for this review is available at the Department of Rheumatology, VU University Medical Center, Amsterdam, the Netherlands. The current report is an update and expansion of the initial protocol (ie, inclusion of high-dose transplantation studies), results of which were published as an abstract. ${ }^{15}$ Delay in completion was caused by personal circumstances.

\section{Information sources}

The search was performed in three databases: MEDLINE, EMBASE and Cochrane Library. Systematic reviews found were scrutinised for relevant citations. This was also carried out in bibliographies of eligible articles.

\section{Search strategy}

A systematic search for published studies was performed. First, the MEDLINE and EMBASE databases (1 January 1995 to September 2012) were searched. The literature search started from 1995, at the time Dual X-ray absorptiometry (DXA) was introduced in patient care. No language restriction was applied, but after screening only manuscripts written in the English language remained. A search of two defined search clusters, termed "osteoporosis' and "glucocorticoids", was carried out. The cluster osteoporosis comprised all citations containing any of the text or thesaurus words "osteoporosis", "osteopenia", "bone density", "bone mass", "densitometry", "absorptiometry" and "fractures" (all trees, all subheadings). Similarly, the cluster "glucocorticoids" comprised all citations containing any of the text words "predniso*", "corticoster*", "glucocort*" (* indicates a wildcard) or thesaurus words "anti-inflammatory agentssteroidal" or "glucocorticoids-synthetic". To obtain all studies on glucocorticoid-induced osteoporosis, the clusters osteoporosis and glucocorticoids were intersected. Second, the Cochrane Library was searched (1995 to 2012). Finally, duplicates were removed. The searches described were carried out with the help of an information specialist of VUmc university library.

\section{Eligibility criteria}

To be included in this review, the following criteria had to be met:

- Patients either had a chronic inflammatory disease or recently underwent a lung, liver, kidney or heart transplantation;

- Bone mass measurements were performed by DXA;

- Report of bone mass at baseline and after 1 year or later.

\section{Selection criteria}

First screen: The title, abstract and keywords of the selected articles were initially screened to exclude animal studies, editorials, letters and reviews, retrospective results and cross-sectional studies, studies employing single measurements, studies employing bone mineral density (BMD) or content measurements other than DXA, and studies without data on either the lumbar spine or femoral neck. For chronic inflammatory disease, studies on diseases or situations that most likely affect bone mass other than chronic inflammatory disease and GC were also excluded. This included Cushing's disease, hypogonadism, hyper (para) thyroidism, chronic liver disease, insulin-dependent diabetes mellitus, renal insufficiency, anorexia, cancer, Addison"s disease and malabsorption syndromes as well as studies in patients $<18$ years of age. For transplantation studies, only the age criterion was applied. Regarding treatment, only vitamin $\mathrm{D}$ and calcium supplementation was allowed; that is, all groups of patients on specific antiosteoporosis treatment were excluded. Any study that appeared relevant to at least one of the two reviewers (MMEB, MB) was retrieved for further scrutiny. The same first screen criteria were then used to further select retrieved articles. 
Second screen: Additional criteria were applied to the articles selected after screening. Studies on non-systemic GC therapy, studies that did not provide a clear description of the amount, kind and duration of GC treatment, and studies not explicitly distinguishing between patient groups that did or did not receive GC therapy were excluded. Similarly, studies not explicitly distinguishing between patient groups that did or did not receive specific antiosteoporotic medication were excluded. No language restriction was applied. Patients with asthma use topical GC at irregular intervals, and such use is suboptimally documented in studies. Therefore, studies including patients with asthma were only included when the percentage of such patients did not exceed $50 \%$.

\section{Outcome measure}

Since GC affect trabecular bone more than cortical bone, ${ }^{1}{ }^{2}$ the primary outcome measure was the 12-month change in lumbar spine bone density $\left(\mathrm{g} / \mathrm{cm}^{2}\right)$ best reflecting trabecular axial bone loss, expressed as a percentage of the initial measurement. The secondary outcome measure was the 12-month change in femoral neck density.

\section{Data extraction}

Where a study population was reported more than once, the most extensive report was used. Data extraction was performed using a standardised form. Means and measures of dispersion were approximated from figures in the manuscript where necessary.

\section{Assessment of study quality}

The application of the selection criteria of the first screen to (1) the title, abstract and keywords and (2) the remaining retrieved articles, and the application of the second screen, subsequent data extraction of the articles selected and assessment of the methodological quality of the eligible cohorts were all performed by two independent reviewers (MMEB, WFL) with standardised forms. Discrepancies were resolved by mutual agreement. Where necessary, a third reviewer (LHDvT) was consulted if disagreement persisted. All assessments were unblinded for authors, institution and journal as the reviewers were familiar with the literature on GC-induced osteoporosis.

\section{Analysis and statistical techniques Data handling and imputation}

Essential data were frequently incompletely reported and needed to be optimised to allow quantitative analysis as follows.

All data were standardised to reflect a 1-year period. The reported measure of the middle of the data (median, weighted median, weighted mean) was assumed to approximate the mean. For sample size, the number of patients completing the study was used where available.
GC exposure was expressed as (or recalculated to) mean daily dose in prednisone equivalents. Where necessary, this was calculated from the cumulative dose. In one study, this dose was approximated from a time period longer than 1 year. ${ }^{16}$ Overall, the average mean dose was calculated from individual study means weighted by sample size. Studies were classified as 'starter' or 'chronic user' depending on the patient population. In some cases, this was difficult: four studies of GC starters included patients starting up to 3 months prior to inclusion. ${ }^{17-20}$ Another study defined chronic GC use as starting at least 1 -month before inclusion. ${ }^{21}$ One very large study reported previous GC use by duration, i.e. as the percentage of patients with a duration $<4$ months, 4-12 months and >12 months before inclusion. ${ }^{22}$ The latter two studies were classified as chronic user studies, whereas the first four were classified as starter studies. In transplant studies, the GC amount related to rejection episode treatments (where reported) was added to the 1-year cumulative dose.

Bone loss was assumed to be linear over time in chronic disease, but not in transplantation settings. Consequently, to estimate 1-year bone loss, interpolation (where necessary) was only allowed in studies of chronic disease. Bone loss in the spine was assumed to be homogeneous, and the actual vertebrae measured were ignored. Bone loss was expressed as a mean percentage of baseline; where necessary, this was calculated by dividing the group mean absolute change by the mean baseline value. In one study, the reported SD of change of $\mathrm{BMD}$ was interpreted to be in fact the $\mathrm{SE}{ }^{23}$

In 15 CID studies (15 for lumbar spine and 12 for femoral neck data) and 4 transplantation studies (4 for lumbar spine and 2 for femoral neck data), the SD of mean bone loss had to be imputed. This was carried out with the model developed by Furukawa: all available SDs and corresponding sample sizes at month 12 were used to calculate a pooled SD which was subsequently entered for the missing SDs. ${ }^{24}$

\section{Meta-analysis and meta-regression}

To estimate bone loss in the two groups at the two sites, meta-analysis was done by applying inverse variance random-effect models by default in order to accommodate the anticipated heterogeneity among study results. ${ }^{25}$ Heterogeneity was studied by funnel plots and $\mathrm{I}^{2}$ statistics. ${ }^{26}$ In addition, several sensitivity analyses were performed in attempts to address heterogeneity (see below).

The relation of bone loss to possible predictors was studied by meta-regression analysis, applying the study weights used in the meta-analysis. These predictors were GC dose (studied separately in chronic disease and transplantation studies); GC start or chronic dosing; and calcium/vitamin D supplementation (the latter two factors studied only in chronic disease, because most patients in transplantation studies received supplementation and were GC starters). In the analysis of GC dose as 
Figure 1 PRISMA flow chart: selection of studies. BMD, bone mineral density.

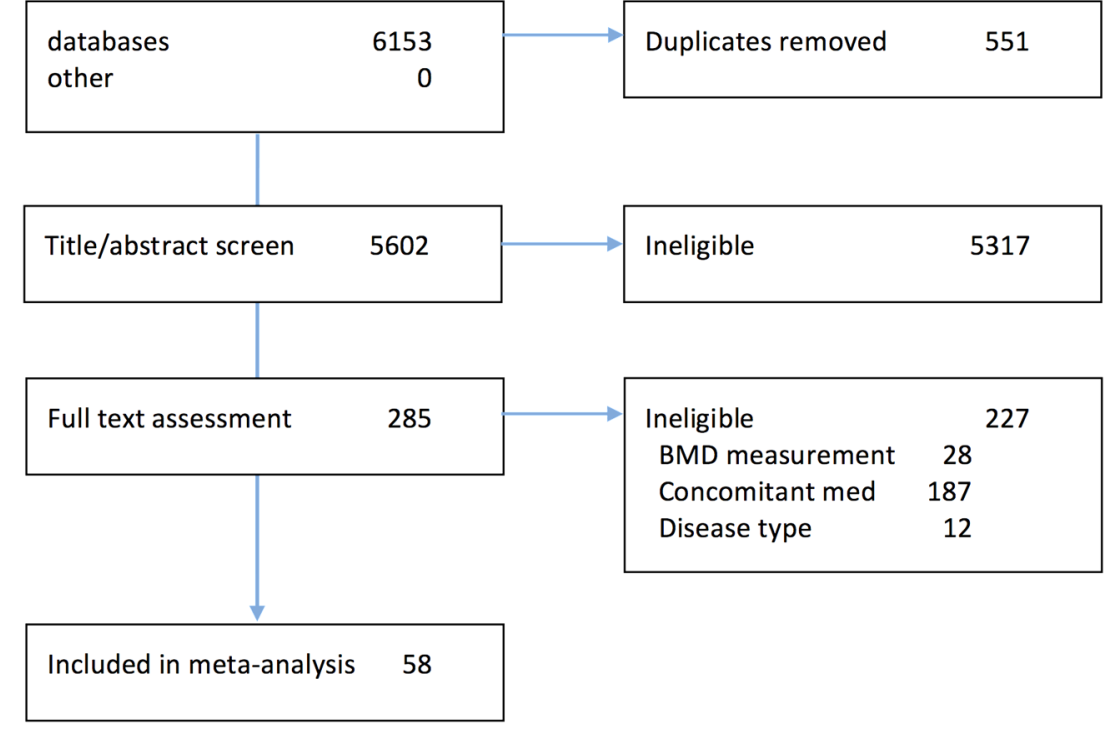

Figure 2 Mean bone loss in studies of patients treated with glucocorticoids. Horizontal lines indicate weighted mean, vertical lines $95 \% \mathrm{Cl}$. BMD, bone mineral density.

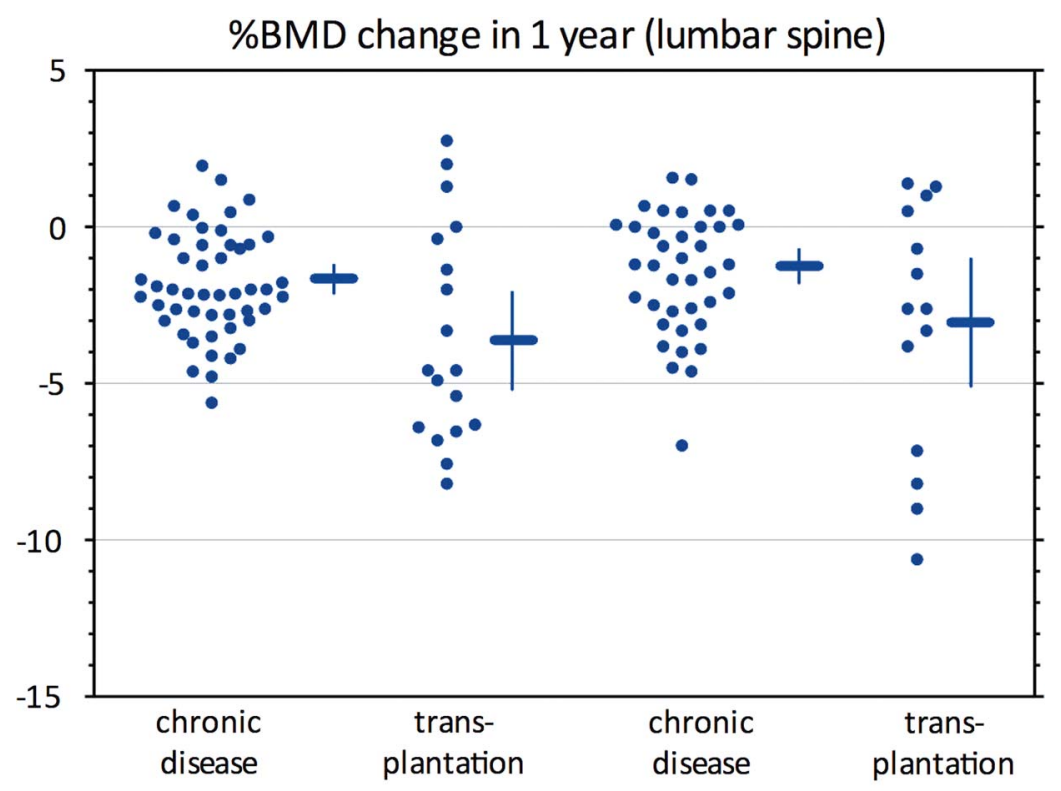

lumbar spine a predictor, GC start/chronic and calcium/vitamin supplementation were additionally studied as potential effect modifiers. In a post hoc analysis suggested during peer review, study year was studied as a predictor in univariate analysis. These meta-regression analyses were performed by the R package metaphor. ${ }^{27}$

\section{Sensitivity analyses}

In both disease groups, bone loss was compared between: (1) Studies with high versus low quality (cut-off: median quality score); (2) studies that reported a measure of variation for the mean result (SD) versus those that did not; and (3) studies with high versus low precision of the bone loss estimate (cut-off: median weight in meta-analysis). Additionally, in the transplantation group, bone loss was compared between kidney and other organ transplantations. In a post hoc analysis suggested during peer review, we checked the relation between the primary outcome measures, relative bone loss (change expressed as a percentage of baseline) and absolute bone loss (change expressed in $\mathrm{g} / \mathrm{cm}^{2}$ ); between baseline BMD and absolute change in BMD; and between baseline BMD and BMD at year 1 .

\section{RESULTS}

\section{Included studies}

The PRISMA flow chart illustrates the selection procedure of studies (Figure 1). The search was initially performed for the period 1995-2010, and later updated to 
Table 1 Characteristics of included studies

\begin{tabular}{|c|c|c|}
\hline & $\begin{array}{l}\text { Chronic Inflammatory } \\
\text { disease }\end{array}$ & Transplantation \\
\hline Cohorts & 49 & 18 \\
\hline RCT arms & 34 & 9 \\
\hline Patients ( $\mathrm{n}, \mathrm{n}$ with follow-up) & 1818,1519 & 635,571 \\
\hline \multirow[t]{4}{*}{ Diagnosis } & 313 & Kidney \\
\hline & SLE & Heart \\
\hline & PMR & Lung \\
\hline & Mixed & Liver \\
\hline Women (\%) & 71 & 34 \\
\hline Mean age (years) & 51 & 46 \\
\hline Starters (\% patients) & 32 & 87 \\
\hline Patients on $\mathrm{Ca} / \mathrm{D}(\%)$ & 67 & 72 \\
\hline Mean GC dose (mg/day) & 9.3 & 15.7 \\
\hline range & $1.2-16.4$ & $6.0-52.7$ \\
\hline \multicolumn{3}{|c|}{ 1-year change in BMD ( $\%$ of baseline) } \\
\hline Lumbar spine mean* & -1.7 & -3.6 \\
\hline $95 \% \mathrm{Cl}$ & -2.2 to -1.2 & -5.2 to -2.0 \\
\hline Femoral neck mean* & -1.3 & -3.1 \\
\hline $95 \% \mathrm{Cl}$ & -1.8 to -0.7 & -5.1 to -1.1 \\
\hline
\end{tabular}

September 2012, and is presented as one search. The first search yielded 4098 citations and the second 1504 , giving a total of 5602 titles. A total of 285 articles were retrieved. After the first and second screening, 225 articles were excluded, among them 14 potentially relevant abstracts in English that turned out to be studies in foreign languages. Data from these abstracts or articles either did not meet the inclusion criteria or did not present relevant data that could be extracted. These studies were therefore not included. Five of the remaining articles were excluded because they showed data presented in other included articles. The bibliographic search of five key reviews and citations of the selected studies added no relevant studies.

The same search strategy was subsequently run again in 2012 (PUBMED from September 2010 to October 2012; EMBASE and Cochrane Library) to capture the most recently indexed studies. This complementary search yielded 1504 new references. Eight articles were retrieved. After screening, all studies were excluded. Thus, 58 articles were left for data extraction and quality assessment (full references in online supplementary appendix 1).

Characteristics of included studies are presented in summary (table 1) and in detail (table 2). In CID, 42 articles yielded a total of 49 cohorts/study arms and 1519 patients (1818 at baseline, ie, $16 \%$ loss to follow-up). Most studies included patients suffering from one CID, with RA as the most frequently studied disease in homogeneous populations $(21 \%)$. In transplantation, 16 articles yielded a total of 18 cohorts/study arms and 571 patients (635 at baseline, ie, $11 \%$ loss to follow-up).
The majority of patients $(79 \%)$ underwent kidney transplantation. Patients with CID were most frequently women of middle age $(72 \%$ females, mean age 56 years); $66 \%$ were chronic GC users. In contrast, most patients with transplant were younger men (64\% males, mean age 46 years); $87 \%$ were GC starters. Calcium and vitamin $\mathrm{D}$ were prescribed in $68 \%$ and $72 \%$ of the patients with CID and patients with transplant, respectively.

\section{Study quality assessment}

An overview of the quality assessment is presented in online supplementary appendix 2 .

All studies presented data on the length of follow-up and age of the study population. Reporting on missing data and loss to follow-up was reported in half of the studies included. For GC use prior to study, the percentage given only applies to chronic user studies. In $63 \%$ of the chronic user studies in which patients already were using GC, the mean dose was reported. Only $38 \%$ of the transplantation studies reported the number of rejection cases and the treatment regimen.

\section{Meta-analysis}

Lumbar spine BMD was measured in all study arms, femoral neck BMD in 51 of 67 arms. Both groups lost bone at both sites; all changes, $\mathrm{p}<0.0001$.

Patients with CID lost less bone than transplantation patients at both sites (Table 1, Figure 2). In the lumbar spine, results were $-1.7 \%(95 \%$ CI $-2.2 \%$ to $-1.2 \%)$ in patients with CID versus $-3.6 \%(-5.2 \%$ to $-2.0 \%)$ in patients with transplant; difference $1.8 \%(-3.1 \%$ to - 
Table 2A Details of included studies on chronic inflammatory disease. Studies are sorted on starter status (A) and transplantation type (B), respectively; and on increasing lumbar spine SD (decreasing weight in meta-analysis). Italics: imputed SDs

\begin{tabular}{|c|c|c|c|c|c|c|c|c|c|}
\hline \multirow[b]{3}{*}{ Author, year } & \multirow[b]{3}{*}{ RCT } & \multirow{3}{*}{$\begin{array}{l}\mathrm{N} \\
\text { (at } 1 \text { year) }\end{array}$} & \multirow[b]{3}{*}{$\mathrm{Ca} / \mathrm{D}$} & \multicolumn{2}{|c|}{ Glucocorticoid } & \multicolumn{4}{|c|}{ Bone loss ( $\%$ of baseline) } \\
\hline & & & & \multirow[b]{2}{*}{ Starter } & \multirow{2}{*}{$\begin{array}{l}\text { Daily dose } \\
\text { Mean }\end{array}$} & \multicolumn{2}{|c|}{$\begin{array}{l}\text { Lumbar } \\
\text { spine }\end{array}$} & \multicolumn{2}{|c|}{$\begin{array}{l}\text { Femoral } \\
\text { neck }\end{array}$} \\
\hline & & & & & & Mean & SD & Mean & SD \\
\hline Ferraccioli, 1996 I & + & 10 & - & + & 1.9 & -2.1 & 1.1 & - & - \\
\hline Ferraccioli, 1996 II & + & 12 & - & + & 5.1 & -4.8 & 1.8 & - & - \\
\hline Jenkins, 1999 & + & 10 & + & + & 8.2 & -3.7 & 2.8 & -1.2 & 3.6 \\
\hline Van Offel, 2001 & + & 10 & + & + & 6.9 & -2.5 & 2.8 & 1.5 & 4.8 \\
\hline Boutsen, 2001 & + & 9 & - & + & 8.9 & -4.6 & 2.9 & -3.1 & 4.1 \\
\hline Cohen, 1999 & + & 57 & + & + & 11.1 & -2.8 & 3.8 & -3.1 & 5.3 \\
\hline Adachi, 1996 I & + & 23 & - & + & 13.5 & -2.6 & 4.1 & - & - \\
\hline Adachi, 1996 II & + & 26 & - & + & 12.6 & -4.1 & 4.1 & - & - \\
\hline Cacoub, 2001 I & + & 34 & + & + & 15.1 & -3.9 & 4.1 & - & - \\
\hline Cacoub, 2001 II & + & 33 & + & + & 16.4 & -3.0 & 4.1 & - & - \\
\hline Dolan, 1997 I & - & 27 & - & + & 10.6 & -2.6 & 4.1 & -4.5 & 4.8 \\
\hline Dolan, 1997 II & - & 23 & - & + & 3.6 & -2.2 & 4.1 & -3.9 & 4.8 \\
\hline Messina, 1992 & + & 8 & - & + & 10.0 & -2.2 & 4.1 & -7.0 & 4.8 \\
\hline Pearce, 1998 & - & 19 & - & + & 6.0 & -2.2 & 4.1 & -2.5 & 4.8 \\
\hline van Everdingen, 2003 & + & 34 & + & + & 10.0 & -2.7 & 4.1 & 0.0 & 4.8 \\
\hline Adachi, 1997 & + & 63 & + & + & 11.3 & -3.2 & 4.8 & -1.7 & 5.3 \\
\hline van Schaardenburg, 1995 & + & 28 & + & + & 9.8 & -3.4 & 5.3 & -4.0 & 4.2 \\
\hline Hansen, 1999 & - & 42 & - & + & 5.9 & -2.7 & 5.8 & - & - \\
\hline Healey, 1996 & + & 22 & + & + & 9.2 & -0.2 & 5.9 & -3.3 & 6.5 \\
\hline weighted mean & & & & & & -3.0 & & -2.7 & \\
\hline Kipen, 1998 & - & 21 & - & - & 11.0 & 0.0 & 1.4 & 0.5 & 1.9 \\
\hline Jardinet, 1999 II & - & 13 & - & - & 5.1 & -0.6 & 1.9 & - & - \\
\hline Buckley, 1996 I & + & 21 & + & - & 5.5 & 0.7 & 2.3 & 0.7 & 2.3 \\
\hline Stoch, 2009 I & + & 13 & + & - & 13.4 & -1.0 & 2.7 & 0.1 & 3.8 \\
\hline Stoch, 2009 II & + & 30 & + & - & 12.6 & -0.6 & 2.7 & 0.1 & 3.8 \\
\hline Hakala, 2012 & + & 65 & + & - & 5.4 & -0.1 & 3.0 & -0.6 & 3.2 \\
\hline Abitbol, 2007 & + & 29 & + & - & 14.0 & -2.0 & 3.2 & -1.7 & 3.7 \\
\hline Buckley, 1996 II & + & 23 & - & - & 5.5 & -1.7 & 3.2 & 0.0 & 3.2 \\
\hline Reid, 2000 & + & 67 & + & - & 13.0 & 0.4 & 3.3 & -0.3 & 4.1 \\
\hline Saag, 1998 & + & 124 & + & - & 10.0 & -0.4 & 3.3 & -1.2 & 4.4 \\
\hline Jardinet, 1999 I & - & 15 & - & - & 12.9 & -2.1 & 3.4 & - & - \\
\hline Westby, 1999 & + & 10 & + & - & 5.0 & -1.9 & 3.5 & -1.4 & 4.9 \\
\hline Geusens, 1998 & + & 13 & + & - & 4.7 & -2.0 & 3.6 & -1.0 & 5.4 \\
\hline Pitt, 1998 & + & 23 & + & - & 6.4 & 0.5 & 3.7 & 1.6 & 6.1 \\
\hline Kotaniemi, 1996 & + & 23 & + & - & 8.4 & -0.6 & 4.1 & -2.7 & 4.8 \\
\hline Lakatos, 2000 & + & 20 & + & - & 14.7 & -4.2 & 4.1 & -2.3 & 4.8 \\
\hline Lems, 2006 & + & 58 & + & - & 7.6 & -1.0 & 4.1 & - & - \\
\hline Loddenkemper, 2003 & - & 51 & + & - & 7.5 & 2.0 & 4.1 & 0.0 & 4.8 \\
\hline Miyamoto, 1999 & - & 44 & - & - & 3.5 & -5.6 & 4.1 & -3.8 & 4.8 \\
\hline Pons, 1995 & - & 21 & - & - & 7.9 & -0.3 & 4.1 & -0.2 & 4.8 \\
\hline Skingle, 1997 & + & 12 & + & - & 8.9 & -1.2 & 4.1 & 0.5 & 4.8 \\
\hline von Tirpitz, 2000 & + & 11 & + & - & 1.2 & -2.2 & 4.1 & - & - \\
\hline Uaratanawong, 2004 & - & 88 & - & - & 11.0 & 0.9 & 4.3 & 0.5 & 4.8 \\
\hline Adachi, 1994 & - & 33 & + & - & 7.8 & -1.8 & 4.4 & - & - \\
\hline Eastell, 2000 & + & 40 & - & - & 5.5 & -2.0 & 4.4 & -1.2 & 3.8 \\
\hline Roux, 1998 & + & 55 & + & - & 11.2 & -2.8 & 4.7 & -2.6 & 5.0 \\
\hline Benucci, 2009 & - & 23 & + & - & 7.5 & -3.0 & 5.3 & -2.4 & 5.3 \\
\hline Cino, 2002 & - & 45 & + & - & 10.5 & -0.7 & 5.4 & -0.6 & 6.7 \\
\hline Sebaldt, 1999 & - & 24 & + & - & 12.6 & -3.5 & 6.4 & -4.6 & 8.2 \\
\hline Hansen, 1998 & + & 14 & - & - & 5.0 & 1.5 & 6.7 & -2.1 & 4.8 \\
\hline weighted mean & & & & & & 1.1 & & -0.8 & \\
\hline
\end{tabular}


Table 2B Details of included studies on transplantation. Studies are sorted on starter status (A) and transplantation type (B), respectively; and on increasing lumbar spine SD (decreasing weight in meta-analysis). Italics: imputed SDs

\begin{tabular}{|c|c|c|c|c|c|c|c|c|c|c|}
\hline \multirow[b]{3}{*}{ Author, year } & \multirow[b]{3}{*}{ Tx } & \multirow[b]{3}{*}{ RCT } & \multirow{3}{*}{$\begin{array}{l}\mathrm{N} \\
\text { (at } 1 \text { year) }\end{array}$} & \multirow[b]{3}{*}{$\mathrm{Ca} / \mathrm{D}$} & \multicolumn{2}{|c|}{ Glucocorticoid } & \multicolumn{4}{|c|}{ Bone loss ( $\%$ of baseline) } \\
\hline & & & & & \multirow[b]{2}{*}{ Starter } & \multirow{2}{*}{$\begin{array}{l}\text { Daily dose } \\
\text { Mean }\end{array}$} & \multicolumn{2}{|c|}{$\begin{array}{l}\text { Lumbar } \\
\text { spine }\end{array}$} & \multicolumn{2}{|c|}{$\begin{array}{l}\text { Femoral } \\
\text { neck }\end{array}$} \\
\hline & & & & & & & Mean & SD & Mean & SD \\
\hline Josephson, 2004 I & Renal & + & 15 & - & + & 22.4 & -2.0 & 5.3 & 1.3 & 7.1 \\
\hline Nowacka, 2001 & Renal & + & 11 & - & + & 14.8 & -0.4 & 5.9 & 1.4 & 11.0 \\
\hline ter Meulen, 2004 & Renal & + & 126 & + & + & 6.0 & 0.0 & 7.3 & -0.7 & 8.4 \\
\hline Wissing, 2005 II & Renal & + & 38 & + & - & 10.4 & -3.3 & 7.4 & -1.5 & 8.0 \\
\hline Casez, 2002 & Renal & - & 30 & - & + & 21.2 & -6.3 & 7.6 & -2.6 & 8.1 \\
\hline De Sevaux, 2000 & Renal & - & 60 & - & + & 17.0 & -6.5 & 7.6 & -3.8 & 8.1 \\
\hline Masse, 2001 & Renal & - & 47 & + & + & 13.6 & -4.6 & 7.6 & - & - \\
\hline Torres, 2004 & Renal & + & 41 & + & + & 17.2 & -5.4 & 7.7 & -2.6 & 8.3 \\
\hline Fan, 1999 & Renal & + & 12 & - & + & 13.4 & -6.4 & 8.0 & -9.0 & 9.6 \\
\hline Yun, 1996 & Renal & - & 19 & - & - & 24.6 & -8.2 & 8.0 & - & - \\
\hline Wissing, 2005 I & Renal & + & 41 & + & + & 10.3 & -1.4 & 8.2 & 1.0 & 7.7 \\
\hline Josephson, 2004 II & Renal & + & 10 & + & + & 22.4 & 1.3 & 10.9 & 0.5 & 3.4 \\
\hline weighted mean & & & & & & & -3.7 & & -1.4 & \\
\hline Berguer, 1994 & Heart & - & 7 & + & + & 11.5 & -4.9 & 5.0 & -8.2 & 6.1 \\
\hline Crawford, 2006 & Liver & + & 25 & + & + & 30.6 & 2.8 & 5.8 & -3.3 & 4.9 \\
\hline Shane, 1998 & Heart & - & 41 & + & + & 24.1 & -6.8 & 6.4 & -10.6 & 7.0 \\
\hline Krieg, 2001 & Heart & - & 17 & + & + & 7.7 & -4.6 & 7.4 & -7.1 & 11.5 \\
\hline Sambrook, 1994 & Heart & - & 13 & - & + & 52.7 & -7.6 & 7.6 & - & - \\
\hline Aris, 2000 & Lung & - & 18 & + & + & 20.2 & 2.0 & 12.7 & - & - \\
\hline Weighted mean & & & & & & & -3.3 & & -7.2 & \\
\hline
\end{tabular}

$0.6 \% ; \mathrm{p}=0003)$. In the femoral neck, results were $-1.3 \%$ $(-1.8 \%$ to $-0.7 \%)$ in patients with CID versus $-3.1 \%$ ($5.1 \%$ to $-1.1 \%)$ in patients with transplant; difference $1.7 \%(3.2 \%$ to $0.1 \% ; \mathrm{p}=0.04)$. In several transplantation studies, patients had more bone loss at 6 months, with partial improvement at 12 months (data not shown). In all meta-analyses, strong evidence of heterogeneity was found, as shown by $\mathrm{I}^{2}$ statistics above $80 \%$ in all analyses, and wide funnel plots (data not shown). There was no evidence of publication bias (Figure 2, top panels).

\section{Meta-regression}

There was no relationship between GC dose and BMD loss in either disease group or bone assessment site (very small $\beta$-coefficients with very wide CIs; figure 3; bottom panels). In univariate analyses, patients with chronic inflammatory disease starting GC appeared to lose more bone in the lumbar spine than chronic users (difference: $1.9 \% \quad(-2.7 \%$ to $-1.0 \%$; $\mathrm{p}=0.003)$, but this did not appear to modify the (lack of) effect of GC dose on bone loss. Calcium/vitamin D supplementation was neither a significant predictor of bone loss in univariate analyses nor an effect modifier.

\section{Sensitivity analysis}

Study quality, availability of precision estimate and precision did not influence the estimate of bone loss. Patients in kidney transplant studies lost less bone in the femoral neck than patients receiving transplants of other organs (difference $5.8 \%$ (2.4\% to $9.1 \% ; \mathrm{p}<0.001)$ ). Year of publication did not influence bone loss in patients with CID, but patients in more recent transplantation studies lost less bone (lumbar spine, $\mathrm{p}=0.002$; femoral neck, $\mathrm{p}=0.004$; see online supplementary appendix 3 ). Correlation between relative and absolute bone loss was excellent $(\mathrm{r}=0.95)$; we found no relationship between BMD at baseline and absolute change in BMD (see online supplementary appendix 4). Consequently, the absolute amount of bone lost was constant over the range of initial BMDs in the studies (see online supplementary appendix 4 ).

\section{DISCUSSION}

This meta-analysis in about 2200 GC users mostly on calcium/vitamin D, but no other antiosteoporotic drugs, shows considerable bone loss in transplantation patients, and modest bone loss in patients with CID. The most obvious explanation for this difference is GC dose, differing by a factor of two. Next, univariate analyses confirmed the finding ${ }^{10}{ }^{11}$ that more bone is lost at the start of GC therapy compared with chronic use, and almost all patients with transplant were GC starters. Further, in CID (and especially RA), GC counteract the effects of systemic inflammation on bone. In contrast, in transplantation, many factors may influence bone mass negatively: ${ }^{28}$ the active underlying disease, particularly in the preoperative period in which the patient is awaiting the 
Figure 3 Composite graph

showing bone loss results by \% weight in the analysis (top panels), and by prednisone dose (bottom panels). In the top indicates the weighted mean per disease group. In the left bottom panel, group symbols correspond to those in the top panels. In the right bottom panel, for each disease group, study results are grouped by quartile of weight: increasing weight. BMD, bone mineral density. panels, a thin horizontal grey line darker colour corresponds to

\%BMD change in 1 year (lumbar spine)
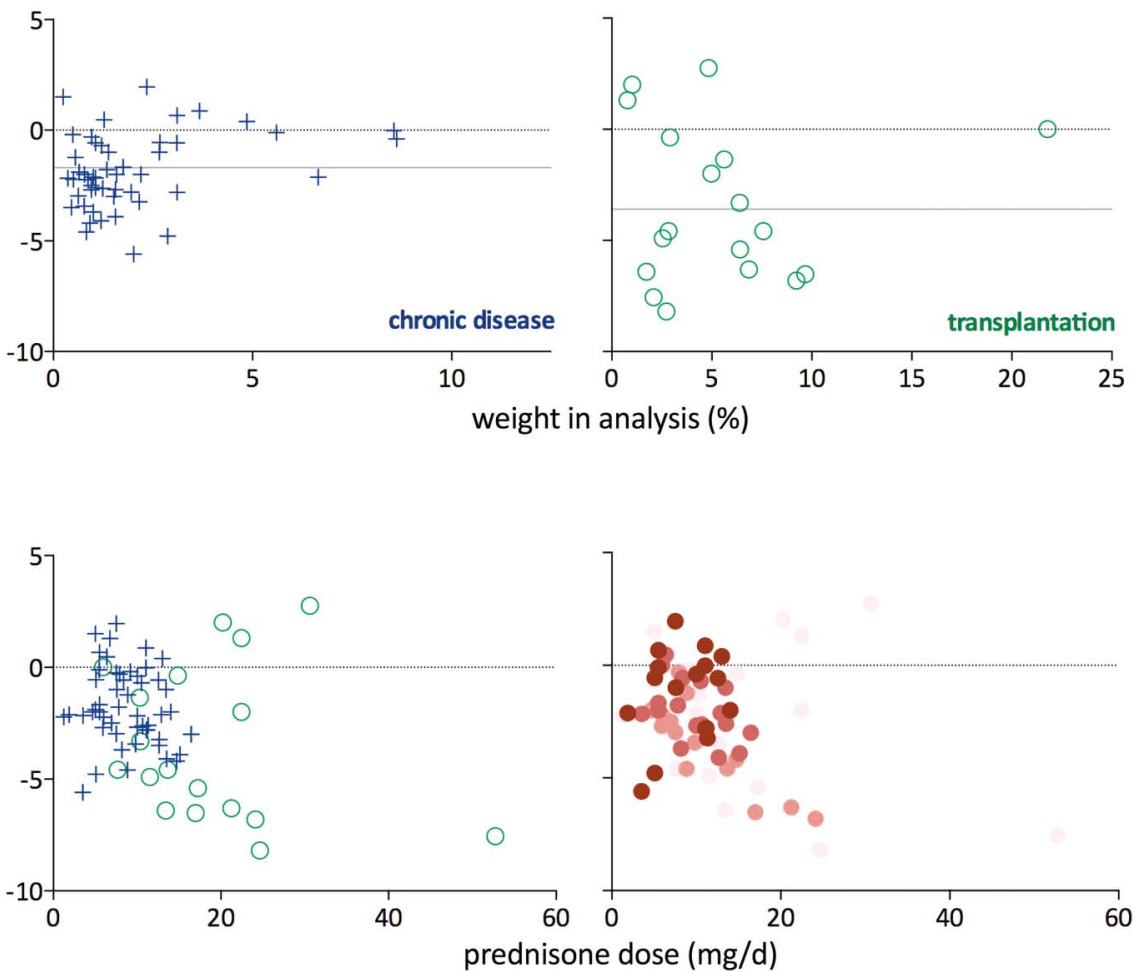

transplantation, the operative procedure itself (malnutrition, infections, immobility, etc) and, in the posttransplant period, immunosuppressive drugs may all have negative effects on the bone (high dose GC, but also cyclosporine and tacrolimus). It is likely that in all three phases, negative effects can disturb the calcium and bone balance.

Another influential factor might be the awareness among rheumatologists of risk of bone loss when prescribing GC to their patients and subsequent lifestyle advice. It should be noted that current treatment of many CID has improved over the past 25 years, reducing disability and improving mobility. Thus, it is likely that bone loss is less in patients on current treatments.

This systematic review is unique, and likely to be the last to report on the relation between pure GC exposure and bone loss, as modern guidelines on GC therapy advocate co-treatment with bisphosphonates or other bone sparing treatments in high-risk patients. ${ }^{12-14}$ However, it is well known that compliance with such guidelines is low, ${ }^{29}$ so that in practice many physicians continue to prescribe GC in high doses or for prolonged periods without offering adequate bone protection. Such non-adherence increases fracture rates. ${ }^{30}$ In clinical settings such as transplantation, part of this lack of compliance may also be explained by the lack of studies proving the benefit of bone protection in that setting. Also, the expectation that bone mass is normal in a young transplantation patient may make bone protection less urgent if the bone loss is felt to be reversible on reduction and withdrawal of GC. Such expectations would need to be backed up by proof, but unfortunately DXA is not a regular feature of a typical pretransplantation workup. ${ }^{31}$ The fact that bone loss seems to decrease in more recent transplantation studies may point to increasing adherence to guidelines. In all, owing to ethical considerations, we expect no new relevant data to be published. Indeed, in our search update for the period 2010-2012, we found no new studies to add to the data set. More recent studies in patients with GC are active comparator studies, for example, RCTs comparing teriparatide to alendronate and risedronate. 3233

This study has limitations. Many study reports were incomplete, forcing us to make a number of assumptions in our calculations of the weighted BMD loss. However, sensitivity analyses did not suggest that such assumptions resulted in bias. It is remarkable that we were unable to identify a doseresponse relationship within the two groups separately. For CID, an explanation could be the narrow range of dosing, mostly between 5 and $15 \mathrm{mg}$ /days. However, this dose range reflects the current state of the art (in fact, with most chronic inflammatory diseases, the range is probably even more narrow in practice) and is wide enough to cover the contentious area above and below $7.5 \mathrm{mg}$ /days, where many physicians feel (without proper evidence) that a tipping point exists between a favourable and unfavourable balance between benefit and harm (see also the recent European League Against Rheumatism subcommittee paper on implementation of existing recommendations ${ }^{34}$ ). For the transplants, possible explanations include disease heterogeneity (different transplant types), power issues related to limited sample size and uncertainty about true levels of GC exposure given a frequent lack of detail on the number of rejection episodes and associated treatment regimens. 
We also did not find a relationship between the use of calcium/vitamin D and bone loss: we suspect that this is the result of the relatively small effect of calcium and vitamin $\mathrm{D}$ on bone loss, and the fact that the majority of patients were supplemented with calcium and vitamin D. Nevertheless, calcium and vitamin D supplementation is advocated in recent guidelines on chronic GC use, based, among others, on two meta-analyses that showed a beneficial effect of vitamin $\mathrm{D}$ plus calcium on BMD versus no supplementation or calcium alone. ${ }^{35} 36$

Large heterogeneity was observed despite the fact that we ordered our analyses according to the two main indications: CID and transplantation. There was a large difference in bone loss between these groups, but within each group no further effect of GC dose on bone loss was observed. This suggests that even in patients treated with high doses of GC, other factors are critical drivers of bone loss. We were only able to detect two such factors: starter versus chronic use in patients with CID, and year of study in patients with transplant. The latter can be regarded as a proxy for changes in treatment regimens that were mostly not recorded in the source studies; somewhat surprisingly, an advantage from new strategies in RA (treat to target, biologics) could not be detected. Apart from incomplete data on dose, especially during the rejection episodes noted above, other likely factors include underlying disease and its activity, comorbidity, age and gender. We excluded diseases most likely to have specific effects on bone mass, but this choice remains somewhat arbitrary. For example, we excluded true malabsoprtion syndromes, but not inflammatory bowel disease, even though such patients can also have malabsorption. Disease activity-not reported in the studies-is especially likely to be a confounder through its effects both on exposure (more active means a higher likelihood of receiving GC and in higher doses) and on outcome (more active means more bone loss). This has been clearly shown in $\mathrm{RA}^{8}{ }^{9}$ but is likely to work in other diseases as well. The effect of menopause on the effect of the underlying disease could not be investigated since most studies did not mention specific data on these factors. Finally, we should caution that in GC-induced osteoporosis there may be a mismatch between fracture risk as determined by bone mass and actual fracture rate. ${ }^{5}$

In conclusion, this meta-analysis provides definitive data on 1-year GC-associated bone loss across a range of diseases and GC doses. In chronic inflammatory diseases, bone loss appears limited and most likely manageable if current antiosteoporotic strategies are fully implemented. In transplantation, and probably also other high-dose settings, bone loss is considerable and represents unmet need. The heterogeneity and lack of further dose effects probably reflects the important influence of other factors, most notably the underlying disease and its treatment by GC.

Competing interests WFL has received fees as speaker and as member of advisory boards: Amgen, Merck and Lilly. LHDvT has a received research grant from Pfizer. MB has received consultation fees from Mundipharma and Pfizer.
Ethics approval Ethics boards of source studies.

Provenance and peer review Not commissioned; externally peer reviewed.

Data sharing statement No additional data are available.

Open Access This is an Open Access article distributed in accordance with the Creative Commons Attribution Non Commercial (CC BY-NC 4.0) license, which permits others to distribute, remix, adapt, build upon this work noncommercially, and license their derivative works on different terms, provided the original work is properly cited and the use is non-commercial. See: http:// creativecommons.org/licenses/by-nc/4.0/

\section{REFERENCES}

1. Canalis E, Mazziotti G, Giustina A, et al. Glucocorticoid-induced osteoporosis: pathophysiology and therapy. Osteoporos Int 2007;18:1319-28.

2. Seibel MJ, Cooper MS, Zhou H. Glucocorticoid-induced osteoporosis: mechanisms, management and future perspectives. Lancet Diabetes Endocrinol 2013;1: 59-70.

3. Curtis JR, Westfall AO, Allison J, et al. Population-based assessment of adverse events associated with long-term glucocorticoid use. Arthritis Rheum 2006;44:420-6.

4. Kanis JA, Johansson $\mathrm{H}$, Oden $\mathrm{A}$, et al. A meta-analysis of prior corticosteroid use and fracture risk. J Bone Miner Res 2004;19:893-9.

5. Van Staa TP, Leufkens HG, Abenhaim L, et al. The use of oral corticosteroids and risk of fractures. J Bone Miner Res 2000;15:993-1000.

6. Bijlsma JW, Boers M, Saag KG, et al. Glucocorticoids in the treatment of early and late RA. Ann Rheum Dis 2003;62:1033-7.

7. Lems WF. Are glucocorticoids harmful to bone in early rheumatoid arthritis? Ann N Y Acad Sci 2014;1318:50-4.

8. Da Silva JA, Jacobs JW, Kirwan JR, et al. Safety of low dose glucocorticoid treatment in rheumatoid arthritis: published evidence and prospective trial data. Ann Rheum Dis 2006;65:285-93.

9. Güler Yuksel M, Allaart CF, Goekoop-Ruiterman YP, et al. Changes in hand and generalised bone mineral density in patients with recent-onset rheumatoid arthritis. Ann Rheum Dis 2009;68:330-6.

10. Teitelbaum SL, Seton MP, Saag KG. Should bisphosphonates be used for longterm treatment of glucocorticoid induced osteoporosis? Arthritis Rheum 2011;63:325-8.

11. Geusens P, Lems WF. Osteoimmunology and osteoporosis. Arthritis Res Ther 2011;13:242.

12. Hoes JN, Jacobs JWG, Boers M, et al. EULAR evidence-based recommendations on the management of systemic glucocortidoid therapy in rheumatic diseases. Ann Rheum Dis 2007;66:1560-7.

13. Grossman JM, Gordon R, Ranganath VK, et al. American Colloge of Rheumatology 2010 recommendations for the prevention and treatment of glucocorticoid-induced osteoporosis. Arthritis Care Res 2010;62:1515-26.

14. Lekanwasam S, Adachi JD, Agnusdei D, et al. A framework for the development of guidelines for the management of glucocorticoid-induced osteoporosis. Ost Int 2012;23:2257-76.

15. Lodder MC, Lems WF, Kostense PJ, et a/Bone loss due to glucocorticoids: update of a systematic review of prospective studies in rheumatoid arthritis and other diseases [abstract]. Ann Rheum Dis 2003;62(suppl 1):94.

16. Geusens P, Dequeker J, Vanhoof J, et al. Cyclical etidronate increases bone density in the spine and hip of postmenopausal women receiving long term corticosteroid treatment. A double blind, randomised placebo controlled study. Ann Rheum Dis 1998;57:724-7.

17. Cohen S, Levy RM, Keller M, et al. Risedronate therapy prevents corticosteroid-induced bone loss: a twelve-month, multicenter, randomized, double-blind, placebo-controlled, parallel-group study. Arthritis Rheum 1999;42:2309-18.

18. Jenkins EA, Walker-Bone KE, Wood A, et al. The prevention of corticosteroid-induced bone loss with intermittent cyclical etidronate. Scand J Rheumatol 1999;28:152-6.

19. Roux C, Oriente P, Laan R, et al. Randomized trial of effect of cyclical etidronate in the prevention of corticosteroid-induced bone loss. J Clin Endocrinol Metab 1998;83:1128-33.

20. van Schaardenburg D, Valkema R, Dijkmans BA, et al. Prednisone treatment of elderly-onset rheumatoid arthritis. Disease activity and bone mass in comparison with chloroquine treatment. Arthritis Rheum 1995;38:334-42.

21. Skingle SJ, Moore DJ, Crisp AJ. Cyclical etidronate increases lumbar spine bone density in patients on long-term glucocorticosteroid therapy. Int J Clin Pract 1997;51:364-7. 
22. Saag KG, Emkey R, Schnitzer TJ, et al. Alendronate for the prevention and treatment of glucocorticoid-induced osteoporosis. Glucocorticoid-Induced Osteoporosis Intervention Study Group. N Engl J Med 1998:339:292-9.

23. Cacoub P, Chemlal K, Khalifa P, et al. Deflazacort versus prednisone in patients with giant cell arteritis: effects on bone mass loss. J Rheumatol 2001;28:2474-9.

24. Furukawa TA, Barbui C, Cipriani A, et al. Imputing missing standard deviations in meta-analyses can provide accurate results. J Clin Epidemiol 2006;59:7-10.

25. DerSimonian R, Laird N. Meta-analysis in clinical trials. Control Clin Trials 1986;7:177-88.

26. Higgins JP, Thompson SG, Deeks JJ, et al. Measuring inconsistency in meta-analyses. BMJ 2003;327:557-60.

27. Viechtbauer $\mathrm{W}$. Conducting meta-analyses in $\mathrm{R}$ with the metafor package. J Stat Software 2010;36:1-48.

28. Kulak CA, Borba VZ, Kulak Júnior J, et al. Transplantation osteoporosis. Arq Bras Endocrinol Metabol 2006;50:783-92.

29. Cramer JA, Gold DT, Silverman SL, et al. A systematic review of persistence and compliance with bisphosphonates for osteoporosis. Osteoporos Int 2007:18:1023-31.

30. Caro JJ, Ishak KJ, Huybrechts KF, et al. The impact of compliance with osteoporosis therapy on fracture rates in actual practice. Osteoporos Int 2004;15:1003-8.
31. Ebeling PR. Transplantation osteoporosis. In: Rosen CJ, ed. Primer on the metabolic bone diseases and disorders of mineral metabolism. 8th edn. Hoboken: Wiley Blackwell, 2013: 495-507.

32. Saag KG, Zanchetta JR, DeVogelaer JP, et al. Effects of teriparatide versus alendronate for testing glucocorticoid-induced osteoporis: 36 months results of a randomized, double-blind, controlled trial. Arthritis Rheum 2009;60:3346-55.

33. Gluer CC, Marin F, Ringe JD, et al. Comparative effects of teriparatide and risedronate in GIOP in men: 18 month results of the EuroGIOPs trial. J Bone Min Res 2013;28: 1365-8.

34. Strehl C, Bijlsma JW, de Wit M, et al. Defining conditions where long-term glucocorticoid treatment has an acceptably low level of harm to facilitate implementation of existing recommendations: viewpoints from an EULAR task force. Ann Rheum Dis 2016;75: 952-7.

35. Amin S, LaValley MP, Simms RW, et al. The role of vitamin D in corticosteroid-induced osteoporosis: a meta analytic approach. Arthritis Rheum 1999;42:1740-51.

36. Amin S, Lavalley MP, Simms RW, et al. The comparative efficacy of drug therapies used for the management of corticosteroid-induced osteoporosis: a meta-regression. J Bone Min Res 2002;17: 1512-26. 